\title{
Constraining the symmetry energy from fragment yields
}

\author{
P. MARINI ${ }^{1}$ and A. Bonasera ${ }^{1,2}$ \\ for the S. J. YENNELLO'S GROUP \\ ${ }^{1}$ Cyclotron Institute, Texas A\&M University, College Station, TX-77843, USA \\ ${ }^{2}$ LNS-INFN, via Santa Sofia 62, 95123 Catania, Italy
}

\begin{abstract}
Methods of extraction of the symmetry energy coefficient to temperature ratio from isobaric and isotopic yields of fragments produced in Fermi-energy heavy-ion collisions are discussed. Consistent results are obtained when the hot fragmenting source is well characterized and its excitation energy and isotopic composition are properly taken into account. The results, independent of the mass number of the detected fragments, suggest that their fate is decided very early in the reaction. A comparison to the Statistical Multifragmentation Model (SMM) predictions is also presented.
\end{abstract}

The effort to understand the properties of asymmetric nuclear matter both at normal densities and at densities away from the saturation density has stimulated a growing interest in isospin effects in nuclear reactions. The isotopic distribution of fragments produced in multifragmentation events in central collisions at intermediate energies can probe properties of low density nuclear matter. Different methods to extract the "symmetry energy" coefficient from yield ratios have been proposed. Among those, we focus on three seemingly different approaches: isoscaling [1-4], m-scaling and isobaric yield ratio method [5], and we show that consistent experimental results can be extracted provided that the properties of the hot fragmenting source, such as its isospin composition and excitation energy, are properly taken into account. We remark that experimentally whether the equilibrium process, occurring during the multifragmentation, takes place at constant pressure or volume (freeze-out hypothesis) is not determined. This ambiguity casts some doubts on the derived quantity, i.e. symmetry energy or symmetry enthalpy. Keeping in mind this ambiguity we will refer to the experimentally extracted quantity as the symmetry energy. 


\section{Isotopic and isobaric yield ratio methods}

It has been experimentally observed that the ratio of the yields of a fragment with $N$ neutrons and $Z$ protons produced in two similar reaction systems with different neutron-to-proton ratios follows an exponential law [1-4]. This relation, known as the isoscaling relation, has been found to describe the measured ratios over a wide range of complex fragments and light particles rather well [1].

Recently, the Modified Fisher Model (MFM) of Ref. [6] has been used to interpret multifragmentation data and extract information on the symmetry energy [5]. An exponential law has been experimentally observed for the ratio of the isotope yields for a fragment, with a given relative isospin asymmetry $m_{f}=\frac{N-Z}{A}$, produced in two similar reactions with different neutron-to-proton ratios [5]. This scaling has been referred to as $m$-scaling.

Isoscaling and m-scaling show significant similarities and to the first order they are found to give an estimation of the same quantity (the so-called isoscaling $\alpha$ parameter) $[5,7]$, provided that Coulomb effects are negligible. Such equivalence was verified and confirmed in our data [7].

The statistical interpretation of isoscaling connects the scaling parameter $\alpha$ to the symmetry-term coefficient $C_{\text {sym }}[1,2]$.

$$
\frac{C_{\text {sym }}}{T}=\frac{\alpha}{4\left[\left(\frac{Z}{A}\right)_{1}^{2}-\left(\frac{Z}{A}\right)_{2}^{2}\right]}=\frac{\alpha}{4 \Delta}
$$

where $\Delta=\left[\left(\frac{Z}{A}\right)_{1}^{2}-\left(\frac{Z}{A}\right)_{2}^{2}\right]$. SMM [2] and the expanding emitting source model [1] identify $\Delta$ as the difference of the asymmetries of the equilibrated emitting sources. Later on we will refer to this quantity as $\Delta_{\text {source }}$. However, due to the phenomenon of fractionation, fragments may not have the same isospin ratio of the fragmenting source. A study of such phenomenon with AMD simulations $[3,8]$ suggested a definition of $\Delta$ which takes into account the proton fraction of the most probable isotope for fragments of a given $Z$ with $A>4$ ("liquid part"). This quantity, referred to as $\Delta_{\text {liquid }}$, relates to the fragments characteristics rather than to those of the two sources, as opposite to $\Delta_{\text {source }}$.

More recently, an investigation of the nuclear phase transition within the Landau Free energy approach [9] suggested $\Delta$ to be function of the average of the fragment relative isospin asymmetry over the events [7]. Such quantity, later referred to as $\Delta_{\left\langle m_{f}\right\rangle}$, takes into account the fragment composition, as well as the event multiplicity. 
Within the MFM [6], a different method to extract the symmetry energy coefficient has been proposed, which does not depend on the definition of $\Delta$. It has been shown that $C_{s y m} / T$ can be extracted as [5]

$$
\frac{C_{\text {sym }}(T)}{T} \approx-\frac{A}{8}[\ln R(3,1, A)-\ln R(1,-1, A)-\delta(3,1, A)]
$$

where $R(I+1, I-1, A)$ are the isotope yield ratios of two pairs of isobars, each differing by 2 units in $I=N-Z$ and produced by the same source.

\section{The analysis}

\subsection{The experiment and the impact of the source reconstruc- tion}

The experiment was performed at Texas A\&M University K500 superconducting Cyclotron. Beams of ${ }^{64,70} \mathrm{Zn}$ and ${ }^{64} \mathrm{Ni}$ at $35 \mathrm{AMeV}$ beam energy were impinged on ${ }^{64,70} \mathrm{Zn}$ and ${ }^{64} \mathrm{Ni}$ targets. The $4 \pi$ NIMROD-ISiS array [10] provided isotopic resolution for $Z \leq 17$. Further details on the experiment can be found in [11].

Particles and events selection were performed to select quasi-projectile (QP) fragmentation events. The QP was reconstructed event by event, requiring an on average spherical event with a detected charge $25 \leq Z \leq 30$. Fragments from non-projectile-like sources were removed. Free neutrons measured by the Neutron Ball were used to correct for the free neutrons emitted by the QP using the procedure discussed in Ref. [12].

Figure 1(a) shows the distribution of the reconstructed QP isospin asymmetry $m_{s}=\frac{N_{s}-Z_{s}}{A_{s}}$ for each of the three systems and for the three systems combined together. The index $s$ refers to the reconstructed QP source. In addition to the shift of the position of the peak of the distribution for the less n-rich system, ${ }^{64} \mathrm{Ni}+{ }^{64} \mathrm{Ni}$, we observe that the distributions' width is large compared to the difference in the average $m_{s}$ between the reacting systems. Fragment yield data was divided according to the relative isospin asymmetry, $m_{s}$, of the reconstructed source in 4 bins, whose boundaries are plotted in Fig. 1(a), and into five bins of transverse excitation energy per nucleon.

A comparison of the isoscaling parameter $\alpha$ extracted from each reaction and for the combined systems when the data was gated on $m_{s}$ showed consistent values within the error bars. A similar behavior was observed for values extracted with the m-scaling. Thus the systems were combined together to increase the statistics. 

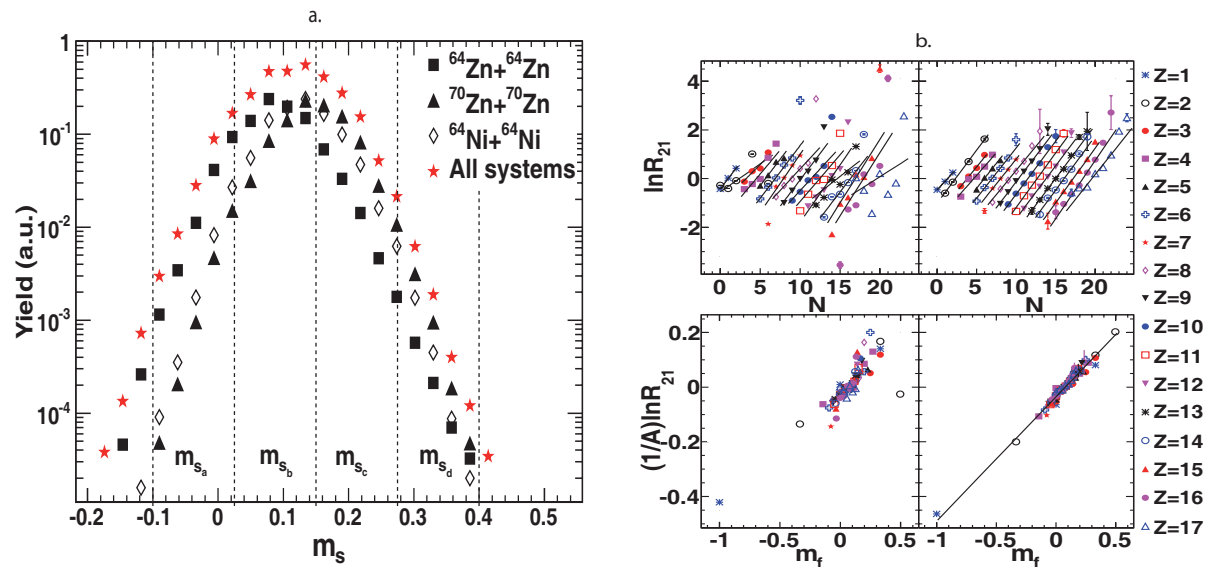

Figure 1: (Color Online) a. Reconstructed QP $m_{s}$ distribution from the different systems and from the combined systems. b. Isoscaling (top) and m-scaling (bottom) plots as obtained comparing two systems $\left({ }^{64} \mathrm{Zn}+{ }^{64} \mathrm{Zn},{ }^{70} \mathrm{Zn}+{ }^{70} \mathrm{Zn}-\mathrm{left}\right)$ and two $m_{s}$ bins of the reconstructed quasi-projectile (right - see text).

In analogy, and extending the work in Ref. [12], we performed both isoscaling and $\mathrm{m}$-scaling between two different $m_{s}$ bins. Significantly better isoscaling and $\mathrm{m}$-scaling were obtained once two sources with different $m_{s}$ rather than two different reactions were selected. This is clearly shown in Fig. 1(b), where isoscaling and m-scaling plots are presented as obtained comparing yields of fragments produced in two different reactions (left panel) and in two sources with different $m_{s}$ (right panel).

\subsection{Comparison of the three methods}

The three different $\Delta$ values $\left(\Delta_{\text {source }}, \Delta_{\text {liquid }}\right.$ and $\left.\Delta_{\left\langle m_{f}\right\rangle}\right)$ as a function of the fragment charge for a given $\Delta \overline{m_{s}}$ and excitation energy combination are plotted in Fig. 2(1). While by definition, $\Delta_{\text {source }}$ (red, dashed) and $\Delta_{\left\langle m_{f}\right\rangle}$ (black, full) are independent of $Z$, the overall behavior of $\Delta_{\text {liquid }}$ shows a rather significant decrease as a function of $Z$, which will cause $\frac{C_{\text {sym }}}{T}$ to increase as a function of $Z$. Structure effects can be recognized in the odd-even behavior of $\Delta_{\text {liquid }}$ (asterisks) for $Z \leq 8$. The large discrepancy between the value of $\Delta_{\text {source }}$ and the values of $\Delta_{\text {liquid }}$ and $\Delta_{\left\langle m_{f}\right\rangle}$ is due to the fact that, while both $\Delta_{\text {liquid }}$ and $\Delta_{\left\langle m_{f}\right\rangle}$ are related to the fragment composition, $\Delta_{\text {source }}$ is related to the source composition.

Figure 2(2) shows a comparison between the three methods, with different choices of $\Delta$, for a given excitation energy (top panels) and for a given 

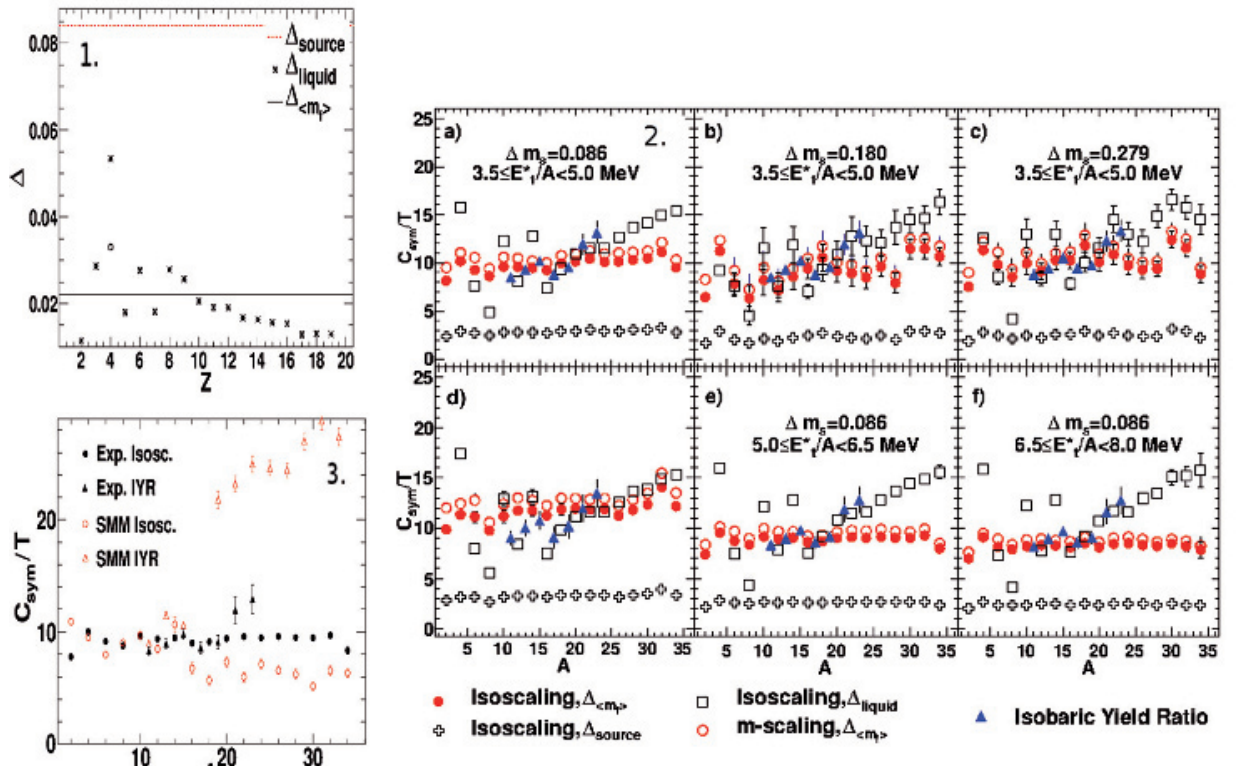

Figure 2: (Color Online) 1. Values of $\Delta$ computed according to the different definitions vs. the fragment charge $Z$ for $\Delta \overline{m_{s}}=0.185$ and $3.5 \leq E_{t}^{\star} / A \leq 5 \mathrm{AMeV}$. 2.Values of $\frac{C_{s y m}}{T}$ obtained with the three different methods. Each panel corresponds to the noted $\Delta \bar{m}_{s}$ and the noted window in $E_{t}^{\star} / A$. Three different choices of $\Delta$ have been used to compute $\frac{C_{s y m}}{T}$ from the $\alpha$ parameter. 3. Experimental (full symbols) and SMM (open symbols) $C_{\text {sym }} / T$ values obtained with isoscaling (circles) and isobaric yield ratio (triangles) methods.

source asymmetry difference ( $a$ and $d-f$ panels). The $\frac{C_{s y m}}{T}$ values obtained by the isobaric yield ratio method (triangles) shows an approximately constant behavior for $A<20$, while an increase is observed for $A=21$ and 23 . We would like to emphasize that one of the main limitation of the isobaric yield ratio method is that it requires a nearly "ideal" isotopic identification, since any contamination by other isotopes to each $(\mathrm{Z}, \mathrm{A})$ fragment affects different isobars by a different amount.

The trends of $\frac{C_{\text {sym }}}{T}$ obtained from the isoscaling and m-scaling depend on the definition of $\Delta$, as already discussed. First, we notice that $\frac{C_{\text {sym }}}{T}$ obtained from the isoscaling with the three definitions of $\Delta$ are not in agreement. Moreover the $\frac{C_{\text {sym }}}{T}$ values determined using $\Delta_{\text {source }}$ (crosses) and $\Delta_{\left\langle m_{f}\right\rangle}$ (circles) do not show any $A$ dependence, while a clearly increasing trend is observed for $A>15$ for values determined using $\Delta_{\text {liquid }}$ (squares). The 
$\frac{C_{\text {sym }}}{T}$ values determined using $\Delta_{\left\langle m_{f}\right\rangle}$ from the isoscaling (full circles) and $\mathrm{m}$-scaling (open circles) show a generally good agreement for all the $\Delta \overline{m_{s}}$ combinations. An excitation energy dependence of the isoscaling and $\mathrm{m}$ scaling extracted $\frac{C_{\text {sym }}}{T}$ can be extrapolated from panels $a, d-f$ independent of the definition of $\Delta$. This trend is in agreement with the excitation energy dependence of the isoscaling parameter $\alpha / \Delta$ observed in [12]. In contrast, a weaker dependence is found for the values extracted with the isobaric yield ratio method.

We now compare the values obtained with the different $\Delta$ definitions to the values extracted from the isobaric yield ratio, since they are independent on the choice of $\Delta$. The $\frac{C_{\text {sym }}}{T}$ values determined using $\Delta_{\text {source }}$ (crosses) are lower than those determined with the isobaric yield ratio method (triangles) by a factor 2 to 3 independent of both $\Delta m_{s}$ and the excitation energy. The $\frac{C_{\text {sym }}}{T}$ values determined using $\Delta_{\text {liquid }}$ (squares) show a rather good agreement only for $A \approx 18-22$ in the central mass region, where the $\Delta_{\text {liquid }} \approx \Delta_{\left\langle m_{f}\right\rangle}$, while it does not reproduce the data for the low mass region. The $\frac{C_{s y m}}{T}$ values determined using $\Delta_{\left\langle m_{f}\right\rangle}$, both from isoscaling and $\mathrm{m}-$ scaling, show good agreement with the isobaric yield ratio values for masses up to $A \approx 20$, for all the $\Delta \overline{m_{s}}$ and excitation energy combinations.

The generally flat behavior of $\frac{C_{s y m}}{T}$ as a function of $A$ (circles and triangles) may be interpreted as the weak influence of secondary decay effects on the observables. Also the constancy of $\frac{C_{s y m}}{T}$ might indicate that the assumption of constant volume is reasonable and we can identify this quantity to the symmetry energy rather than the enthalpy [7].

\subsection{Comparison to SMM predictions}

Many aspects of multifragmentation in nucleus-nucleus collisions have been well described by the SMM [13], which has been widely used to extract information on the symmetry energy [2]. Extensive SMM calculations were preformed varying the size, the excitation energy and $C_{s y m}^{i n}$ of the primary source.

In Fig. 2(3) the experimental (full symbols) and calculated (open symbols) $C_{\text {sym }} / T$ obtained by the isoscaling (circles) and the isobaric yield ratio (triangles) methods are plotted as a function of the fragment mass $A$. The values predicted by SMM have been obtained for $C_{\text {sym }}^{\text {in }}=14 \mathrm{MeV}$ and $E^{\star}=5 \mathrm{MeV} /$ nuc, which gives the best agreement to the experimental data. A value of $C_{s y m}^{i n} \simeq 14 \mathrm{MeV}$ is in agreement with previous experimental results [4]. The predicted (open circles) and experimental (full circles) 
values obtained by isoscaling method are in good agreement, especially for masses $A<16$, for which the de-excitation mechanism implemented in SMM changes [13]. We observe that SMM reproduces the experimental trend and the values. This is not true for the values obtained with the isobaric yield ratio method (triangles), especially for masses $A>16$. This is due to the fact that the isobaric yield ratio method depends much stronger on secondary de-excitation effects than the isoscaling. Therefore it is more sensitive to the secondary de-excitation procedure implemented in the model.

\section{Conclusions}

Three methods to extract the symmetry energy coefficient from fragment yields were compared and their strengths and weaknesses pointed out. Improved isoscaling and $\mathrm{m}$-scaling were observed when selecting quasiprojectile $m_{s}$ bins for n-rich and n-poor systems, while isobaric-yield-ratioextracted values were only slightly affected. Indications that the connection between $\alpha$ and $\frac{C_{\text {sym }}}{T}$ has to be found in the average fragment isotopic asymmetry were presented. The generally flat trend of the extracted symmetry term as a function of $A$ for the mass region with the best mass resolution may be consistent with the lack of secondary de-excitation effects in our data, within our isotopic resolution, as well as with the assumption of a freeze-out volume where the disassembly occurs.

A comparison to SMM predictions indicates $C_{\text {sym }}=14 \mathrm{MeV}$ as the most suitable value to reproduce our data.

This work was supported by the U.S. DOE grant DE-FG03-93ER40773 and the Robert A. Welch Foundation grant A-1266.

\section{References}

[1] Tsang M. B. et al., Phys. Rev. C, 64, (2001) 054615

[2] Botvina A. S. et al., Phys. Rev. C, 65, (2002) 044610

[3] Ono A. et al., Phys. Rev. C, 68, (2003) 051601(R)

[4] Le Fèvre A. et al., Phys. Rev. Lett., 94, (2005) 162701 and Refs. therein

[5] Huang M. et al., Phys. Rev. C, 81, (2010) 044620, 044618 and Nucl. Phys. A 847, (2010) 233

[6] Minich R. W. et al., Phys. Lett., B118, (1982) 458

[7] Marini P. et al., accepted for publication Phys. Rev. C (2012)

[8] Ono A. et al., Phys. Rev. Lett., 68, (1992) 2898 
[9] Tripathi R. et al., arXiv 1010 (2010) 2227

[10] Wuenschel S. et al., Nucl. Instr. Meth. A, 604, (2009) 578 and AIP Conf. Proc., 1099 (2009) 816

[11] Kohley Z. Ph.D. Thesis, Texas A\&M University (2010)

[12] Wuenschel S. et al., Phys. Rev. C, 79, (2009) 061602(R), Nucl. Phys. A, 843 (2010) 1 and Ph.D. Thesis, Texas A\&M University (2009)

[13] Bondorf J. P. et al., Phys. Rep., 257, (1995) 133 and Botvina A. S. et al, Phys. Rev. C, 63 (2001) 061601(R) 